\title{
Nutrition et santé : une attente du consommateur, un objectif de l'industrie laitière
}

\author{
D Cochet \\ Prospérité fermière, 51-53 avenue Fernand Lobbedez, BP 946, 62033 Arras Cedex, France
}

lait / protéine / nutrition / marché

Nutrition and health: a consumer expectation and a goal for the dairy industry.

milk / protein / nutrition / market

UNE ATTENTE DU CONSOMMATEUR

\section{Produits «nutritionnels" : mouvement de fond ou phénomène de mode?}

II semble aujourd'hui admis que la consommation de produits nutritionnels corresponde à une tendance de fond.

L'attitude du consommateur est de plus en plus guidée par l'objectif d'être bien portant et en pleine forme. II est ainsi persuadé que s'il s'alimente bien, il vivra longtemps et que son «look corporel» conditionne le succès de sa vie sociale.

Ceci est le résultat de campagnes d'informations menées déjà depuis quelques années par les milieux médicaux et les médias pour apprendre aux consommateurs qu'il existe des relations entre la qualité de ce qu'ils mangent et certaines maladies ou certaines déficiences nutritionnelles.
Le consommateur sait ainsi, par exemple, qu'il existe une relation étroite entre la matière grasse et les maladies cardio-vasculaires. De même, sa vie sociale a changé. Elle est devenue plus conviviale. II a plus de temps libre qu'il consacre à des activités de loisirs : ce sont bien souvent des activités de détente et des activités sportives qu'il pratique en groupe.

Ces modifications du comportement du consommateur influencent favorablement le développement des produits à forte connotation nutritionnelle.

Le taux de pénétration des produits nutritionnels parmi les produits dits conventionnels est cependant très variable d'une catégorie de produit à une autre et d'un pays à un autre. Le taux de pénétration des crèmes glacées est d'environ $1 \%$, alors que celui des produits laitiers est voisin de $14 \%$.

En France, on constate ainsi que les produits allégés et diététiques représen- 
tent déjà un marché de $20.10^{9}$ et que ces nouvelles préparations pourraient représenter jusqu'à $10 \%$ de leur marché de référence en l'an 2000.

La France et les pays d'Europe moyenne se situant entre 2 extrêmes. Au Nord, les pays scandinaves et particulièrement la Suède où la part des produits nutritionnels représente $10 \%$ de la consommation alimentaire et au Sud, les pays méditerranéens avec moins de $1 \%$ de produits nutritionnels.

Dans le domaine de la santé, comme le montre le tableau I, la consommation des ménages est en augmentation et son poids dans la consommation totale est en progression régulière.

L'INSEE prévoit d'ailleurs que la santé sera le poste de dépenses le plus important pour les Français à l'horizon 2000 et qu'il pourrait même dépasser le poste alimentaire.

II s'agit bien d'un mouvement de fond et le lait, et plus particulièrement les protéines laitières, peuvent jouer un rôle essentiel dans notre alimentation moderne. Leurs propriétés nutritionnelles en font des ingrédients de choix dans les produits diététiques et de santé destinés aux populations vulnérables et malades.

\section{PROTÉINES LAITIÈRES : DE NOMBREUX SECTEURS D'APPLICATIONS}

\section{Quelles perspectives de marché ?}

Nous pouvons essayer de passer en revue les marchés des produits nutritionnels et de santé où sont aujourd'hui valorisées les protéines laitières et tenter ensuite de faire quelques prévisions.

\section{Les produits allégés}

On ne peut pas parler de produits nutritionnels sans évoquer les produits allégés. Cette vogue de l'alimentation allégée apparaît comme un phénomène durable parce qu'il répond à une attente profonde du consommateur pour des produits sains qui garantissent la forme.

Ces produits apportent une diminution significative du nombre de calories par rapport aux produits conventionnels. Ils sont plus exactement appelés par nos voisins anglosaxons : Low calorie foods. Ce sont des produits qui garnissent tous les jours un peu plus nos «linéaires" avec des «claims» Light, allégé, léger.

Tableau I. Consommation totale des ménages en services médicaux et de santé. Total household expenses in medical and health services

\begin{tabular}{lrrrrr}
\hline & 1982 & 1983 & 1984 & 1985 & 1986 \\
\hline $\begin{array}{l}\text { En } 10^{9} \mathrm{~F} \\
\text { Évolution en \% }\end{array}$ & 170 & 195 & 219 & 246 & 268 \\
$\begin{array}{l}\text { Poids dans la consommation } \\
\text { totale des ménages }\end{array}$ & $+14,6$ & $+15,0$ & $+12,3$ & $+12,0$ & $+9,0$ \\
\hline
\end{tabular}

Source : INSEE 
Certains sont allégés en calories glucidiques; ces produits font appel à de nouveaux ingrédients maintenant bien connus tels que les édulcorants, les polycols, les polydextroses et les fibres.

D'autres sont allégés en calories d'origine lipidique et ont été mis au point grâce à l'arrivée des substituts de matière grasse. On dispose aujourd'hui de 3 grandes catégories de substituts de matières grasse : les substituts à base d'amidon, les substituts à base de protéines et les produits de synthèse. Ce sont les propriétés fonctionnelles des protéines qui sont exploitées pour leur rôle "lubrifiant" dans le cas des substituts à base de protéines.

Trois produits sont en cours de développement :

- l'un à base de protéines de lait et/ou de protéines d'œuf. C'est le produit Simplesse de Monsanto;

- l'autre est aussi un complexe albumine d'œuf et protéines de lait mis au point par Kraft : Trailblazer;

- le troisième est un substitut de matière grasse uniquement à base de protéines de lait mis au point par l'entreprise laitière Land'O Lakes : il s'appelle Galileo.

N'oublions pas les caséinates et les concentrés de protéines sériques qui contribuent largement au développement de certaines spécialités allégées grâce à leur pouvoir émulsifiant et à leur capacité de rétention d'eau. Les principaux secteurs d'application sont la charcuterie, les sauces, et les produits laitiers.

Je pense que les protéines laitières n'ont pas encore tout dit dans le secteur des Fat substitutes et leurs nombreuses propriétés technologiques n'ont certainement pas encore été toutes exploitées. Ainsi, la $\beta$-lactoglobuline est certainement promise à un brillant avenir dans ce marché.

\section{Les produits de la forme}

Toujours dans cette tendance de fond pour des produits de la forme, mais dans le cadre de besoins plus occasionnels que les produits allégés; il existe un marché qui est celui des produits de l'effort et de l'amaigrissement. Ce marché, principalement représenté par des préparations hyperprotéinées à base de caséinates et de protéines totales de lait, est en progression de $15 \%$ par an en Europe (tableau II).

Ces produits sont principalement consommés par 2 catégories de consommateurs. D'une part, ceux qui souhaitent traiter un problème de surpoids et qui font donc appel à des substituts de repas riches en protéines laitières $(41-69 \%$ des français suivent un régime alimentaire, source INSEE), d'autre part, ceux qui souhaitent entretenir ou reconstituer leur masse musculaire dans le cadre d'efforts physiques (tableau III).

Ce marché en croissance régulière risque malheureusement de s'essouffler d'ici quelques années étant donné la concurrence des produits allégés en calories et de certains nouveaux produits enrichis en protéines.

Les caséinates ont été utilisés très régulièrement dans ces préparations hyperpro-

Tableau II. Consommation de produits de l'effort et d'amaigrissement en Europe.

Consumption of products for energizing or slimming diets in Europe.

\begin{tabular}{lrrr}
\hline & 1980 & 1985 & 1988 \\
& & & \\
Volume $(t)$ & 3771 & 4890 & 5260 \\
Valeur $\left(10^{6} \mathrm{~F}\right)$ & 291 & 508 & 728 \\
\hline
\end{tabular}

Source : Prospérité Fermière 
Tableau III. Personnes pratiquant une activité physique en France.

Persons practising a physical activity in France.

\begin{tabular}{cc}
\hline Femmes & Hommes \\
\hline $71 \%$ & $77 \%$ \\
\hline
\end{tabular}

Source INSEE.

téinées depuis leur origine. Depuis 2 ans cependant, les opérateurs comme Weider ont lancé des produits contenant de nouveaux ingrédients laitiers tels que les concentrés de protéines de lactosérum et les concentrés de protéines totales de lait (fig 1). Leur taux d'incorporation est en progression constante bien que restant encore très limité.

Les principaux consommateurs sont les Allemands. L'Allemagne représente près de la moitié du marché où sont présentes toutes les grandes marques telles que $\mathrm{Ha}$ leko, Nesfit, Sanofi, Milupa.
Je complèterai ma présentation sur les produits de la forme en parlant des produits cosmétiques qui répondent à une attente très proche de celle des produits de la forme. Forme et beauté font en effet partie du même univers chez le consommateur.

Si ce sont les femmes qui ont lancé l'histoire de la cosmétique, ce sont les hommes qui, aujourd'hui assurent le développement. Le colostrum et les hydrolysats de protéines trouvent des débouchés très valorisants mais la compétition avec les autres sources protéiques et, en particulier, les protéines végétales est très vive, le végétal ayant une image très positive en cosmétique.

\section{Les laits infantiles}

Ce sont les laits infantiles qui ont le mieux et le plus rapidement exploité les découvertes scientifiques dans le secteur des protéines laitières et notamment avec l'arrivée des concentrés de protéines sériques et des hydrolysats de caséines et de pro-
44 \% Lait écrémé

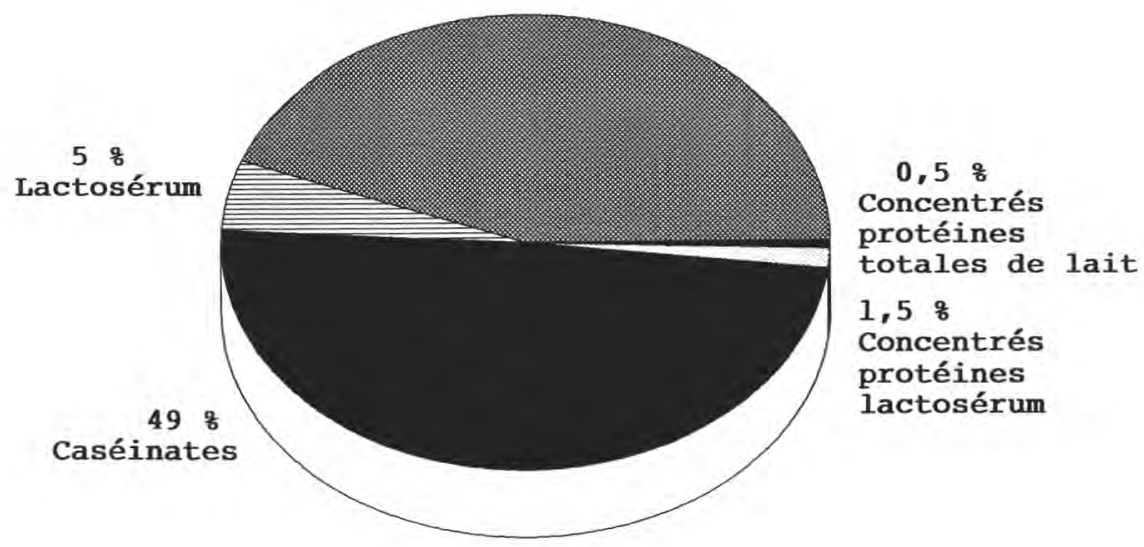

Fig 1. Consommation d'ingrédients laitiers en diététique grand public en volume (année 1988) (source Prospérité Fermière).

Consumption of dairy ingredients in common dietetics, in volume (year 1988). 
téines de lactosérum. L'hydrolyse des protéines de caséine et de lactosérum réduisant l'allergénicité de ces protéines.

Ainsi, les formules ont évolué progressivement des laits maternisés dans les années 1970, aux laits adaptés dans les années 1980 puis aux laits spécifiques et plus particulièrement aux laits hypoallergéniques dans les années 1985.

Le marché des laits infantiles en Europe est relativement stable depuis quelques années et le phénomène le plus intéressant concerne la progression rapide des laits spécifiques et, par conséquent, l'utilisation croissante de concentrés de protéines de lactosérum et d'hydrolysats de caséine et de protéines sériques.

\section{Alimentation de la femme enceinte et allaitante}

Au cours de la grossesse et de la période d'allaitement, les besoins en protéines, en fer et en calcium de la femme augmentent d'environ $10-30 \%$. Naturellement, I'organisme de la femme s'adapte pour assurer ces besoins supplémentaires. Ces adaptations sont cependant encore mal connues et des apports supplémentaires en protéines, en fer et en calcium permettent de mettre la femme enceinte et la femme allaitante à l'abri de toutes déficiences.

Les produits laitières, les phosphopeptides et la lactoferrine sont des ingrédients tout désignés pour assurer ces supplémentations.

\section{La nutrition entérale et parentérale}

Mais c'est dans le marché des produits de nutrition entérale et parentérale que les propriétés nutritionnelles des protéines laitières et plus exactement des hydrolysats de protéines laitières trouvent leur meilleure valorisation.

Je rappelerai brièvement ce qu'est la nutrition entérale et parentérale : elle concerne l'alimentation de patients atteints d'insuffisance des fonctions digestives, les opérés, les malades psychiatriques ainsi que les personnes présentant des problèmes de mastication.

C'est un marché en pleine progression dans les 5 grands pays d'Europe du Nord: $6 \% / a n$ en volume et $12 \% /$ an en valeur (tableau IV). Les situations sont très différentes d'un pays à l'autre. Ainsi, la France est considérée comme le premier marché en Europe avec environ 280.106 $\mathrm{F}$ alors que le marché britannique est de $94.10^{6} \mathrm{~F}$.

Les ingrédients laitiers qui dominent ce marché sont les caséinates, les concentrés de protéines de lactosérum et le lait écrémé (fig 2) mais les meilleures perspectives de développement reviennent aux peptides, au lait à lactose hydrolysé ainsi qu'aux concentrés de protéines totales de lait.

Rappelons que les peptides du lait, et plus particulièrement, les di- et tri-peptides ont l'avantage nutritionnel d'être exploitables dans les cas pathologiques d'atteinte de la muqueuse intestinale et ont toutes les chances de se développer dans les prochaines années.

Tableau IV. Consommation de solutions entérales en Europe.

Consumption of enteral solutions in Europe.

\begin{tabular}{lrrr}
\hline & 1980 & 1985 & 1988 \\
\hline Volume $\left(10^{6} \mathrm{I}\right)$ & 17,5 & 22,6 & 27,7 \\
Valeur $\left(10^{6} \mathrm{~F}\right)$ & 258,5 & 451,0 & 653,0 \\
\hline
\end{tabular}

Source : Prospérité Fermière 


\section{3 \& Caséinates}

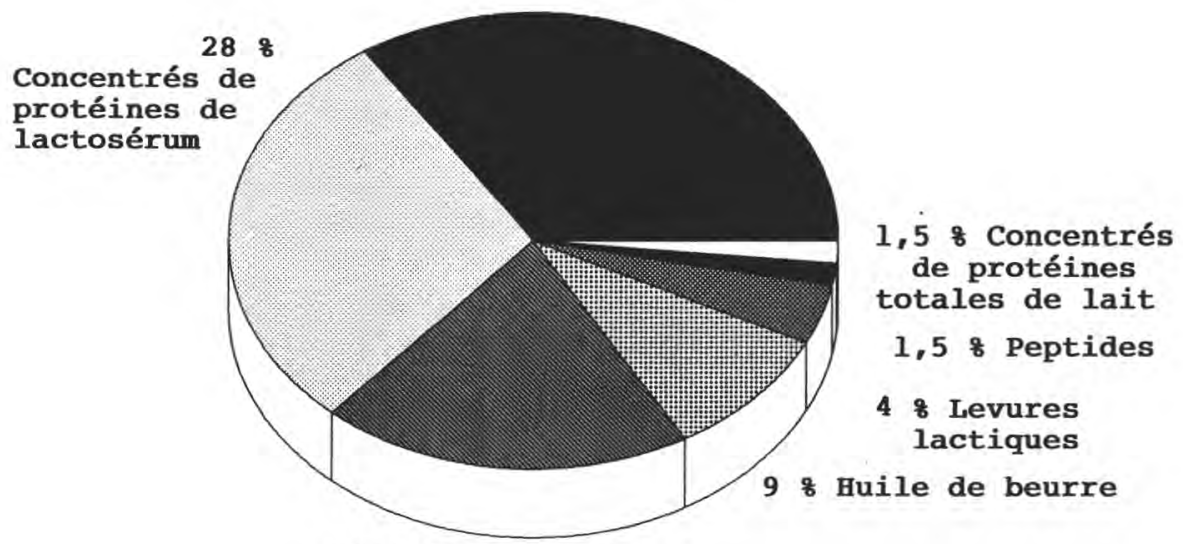

19 \& Lait en poudre

Fig 2. Consommation d'ingrédients laitiers dans les solutions entérales en volume (année 1988) (source Prospérité fermière).

Consumption of dairy ingredients in enteral solutions, in volume (year 1988).

Nous avons vu comment les marchés des produits diététiques exploitaient les propriétés technologiques, mais surtout nutritionnelles, des protéines laitières et de leurs hydrolysats.

Passons maintenant aux propriétés thérapeutiques de ces protéines laitières.

Les activités biologiques de certains peptides du lait laissent espérer des développements dans le domaine du médicament. Des patients atteints de maladies graves pourraient bénéficier des effets thérapeutiques de certaines fractions peptidiques.

Citons ici les principaux effets thérapeutiques déjà démontrés :

- les phosphopeptides résultant de l'hydrolyse de la caséine permettent d'améliorer l'absorption du calcium et aussi d'obtenir des solutions riches en calcium;

- les hydrolysats pepsiques de caséine $\beta$ qui auraient une activité opiacée;
- de même, des peptides issus de l'hydrolyse trypsique de la caséine auraient une activité antihypertensive grâce à l'inhibition de l'angiotensine Enzyme.

Signalons pour finir, l'activité immunomodulante des immunoglobulines et bactériostatique de la lactoferrine et de la lactoperoxydase qui ont déjà trouvé des applications très intéressantes dans le secteur vétérinaire.

\section{La nutrition et la santé animales}

Ceci me permet de faire la transition avec le marché de la nutrition et de la santé animales dans lequel certaines protéines laitières peuvent trouver des valorisations tout aussi intéressantes que dans le marché de la santé humaine.

N'oublions pas nos chers petits compagnons qui souffrent fréquemment des 
mêmes maux que leurs maîtres. Cependant, les perspectives de ventes en volume et en marge sont probablement moins attrayantes que le marché de la nutrition et de la santé humaine.

II serait très prétentieux de croire que les protéines laitières vont révolutionner le marché de la diététique et de la santé.

II est aussi très risqué de faire des prévisions quant au volume de protéines et de peptides commercialisables à 10 ou 20 ans, étant donné que l'on ne connaît pas encore totalement les modes de fonctionnement de ces produits, que leur prix de revient conditionnera leur compétitivité face à des produits de synthèse obtenus par génie génétique ou d'autres protéines d'origine végétale ou animale.

Cependant, on peut faire quelques prévisions à moyen terme à partir de l'évolution passée du phénomène et en tenant compte de l'avis de certains experts.

Ainsi, un accroissement de la consommation de protéines laitières de 1-1,5\%/an en Europe d'ici l'an 2000 semble très réaliste.

\section{Quelles stratégies de développement pour les entreprises laitières}

A l'heure des quotas laitiers et alors que les perspectives de prix de marchés pour les produits laitiers ne sont pas encourageantes, les entreprises laitières doivent inéluctablement envisager de nouvelles stratégies d'avenir, découvrir de nouveaux créneaux, peut-être moins gourmands en volume mais susceptibles de dégager de plus fortes valeurs ajoutées.

Les marchés des produits nutritionnels et de santé représentent aujourd'hui une des voies de diversification les plus sûres et les plus prometteuses :
- d'une part, parce que ces marchés ne sont pas encore arrivés à mâturité,

- d'autre part, parce qu'ils apportent une valorisation très supérieure à celle observée sur les marchés des produits conventionnels.

En France, et plus généralement en Europe, les consommateurs commencent à avoir une vision nutritionnelle et pharmacologique de la nourriture. Cette situation devrait bénéficier aux alliances qui vont se développer entre les sociétés alimentaires et les sociétés pharmaceutiques. Ces alliances auront des conséquences stratégiques à long terme pour les entreprises alimentaires.

II existe déjà aujourd'hui des exemples et, tous les jours, ce phénomène s'amplifie.

Je voudrais commencer par citer l'exemple du groupe Nestlé qui, au début du siècle était une entreprise laitière fabriquant des laits condensés, qui domine maintenant le marché de l'alimentation infantile en Europe, qui possède des sociétés dans le secteur pharmaceutique et qui vient de créer avec Baxter la société Clintec aux USA, spécialisée dans la nutrition entérale. On peut prendre aussi l'exemple de Nutricia, entreprise laitière fondée dans les années 1910 qui est aujourd'hui leader incontesté du marché de la nutrition entérale en Europe.

Plus près de nous, en France, il y a quelques entreprises laitières qui ont certainement compris cette nécessité de s'allier à des entreprises du secteur pharmaceutique et qui ont conclu des accords avec des entreprises du secteur de la santé. Citons :

- la Laitière d'Isigny qui fabrique sous contrat les laits infantiles de la société Wyeth Byla;

- Armor Protéines qui collabore à la fabrication d'un aliment de réanimation pour la société Sopharga; 
- Wyeth Byla et Candia qui ont créé une société commune de commercialisation des laits infantiles en GMS: Sodilac.

Mais c'est au Japon que ce mouvement est le plus fort et il me semble qu'il y a quelques enseignements à tirer de ce qui se passe à l'extrême est de notre continent.

Ainsi Meiji Seika, $n^{\circ} 1$ de la confiserie au Japon est devenu le premier groupe pharmaceutique du pays en commençant sa démarche de diversification après la guerre. II vient de lancer un néosucre grâce à la mise en commun des travaux de recherche et développement des équipes du secteur alimentaire et du secteur pharmaceutique; Ajinomoto, le leader mondial des acides aminés qui en 10 ans a déjà sorti 3 médicaments; Kirin, le géant de la bière est sur le point de lancer ses premiers antibiotiques.

La véritable force et la grande originalité du secteur "Recherche et développement» alimentaire au Japon tient surtout à sa diversification orientée vers la pharmacie.

Le vrai succès de cette diversification ne viendrait-il pas de la mise en commun des efforts de recherche des entreprises alimentaires et des entreprises pharmaceutiques?

Si l'on examine les dépenses de recherche dans l'un et l'autre des secteurs alimentaires et pharmaceutique (tableau V), on s'aperçoit que les entreprises agroalimentaires ont encore de gros efforts à faire pour se mettre au niveau de leurs confrères du secteur pharmaceutique.

On peut ainsi se poser la question : n'est-il pas déjà nécessaire dans un premier temps d'accroître les budgets globalement alloués à la recherche dans le secteur agro-alimentaire?

La taille réduite des entreprises est certainement un frein. On peut espérer que
Tableau V. Dépenses de recherche et développement dans l'industrie agro-alimentaire et pharmaceutique (Valeur moyenne en \% du CA)

$R$ \& $D$ expenses in the food and in the pharmaceutical industry (average value in \% of turnover)

\begin{tabular}{ccc}
\hline $\begin{array}{c}\text { Industrie } \\
\text { agro-alimentaire }\end{array}$ & $\begin{array}{c}\text { Industrie } \\
\text { pharmaceutique }\end{array}$ \\
\hline France & Japon & \\
\hline $0,12 \% \quad 0,63 \%$ & $9 \%$ \\
\hline
\end{tabular}

les récents regroupements qui se sont opérés dans la filière laitière vont permettre d'accroître les ressources des entreprises laitières et ainsi leur puissance de recherche.

Les instituts de recherche publique ne seraient-ils pas des lieux privilégiés pour conduire les recherches communes au secteur alimentaire et au secteur pharmaceutique?

Beaucoup de questions qui pourront être débattues demain en table ronde. Les entreprises laitières françaises ne semblent pas en retard dans ce mouvement de concentration et d'alliance avec des entreprises pharmaceutiques. On ne pourrait aujourd'hui que les encourager à persévérer.

\section{RÉFÉRENCES}

CPCIA (1989) Qualification et valorisation industrielle des protéines du lait. Étude CPCIA, Paris

Eveno R (1990) Les allégés prennent du poids. Usine Nouv 2274, 31

Langley-Danysz P (1989) Lacto-protéines : le lait anti-microbien? Rev Ind Agro-Alim 423, 44-47 
Millet $P$ (1989) Les édulcorants jouent sur tous les tableaux. Stratégies Gourmandes, maijuin, 22-25

Moyal MF (1988) Dietetics in the 90s. Role of the Dietician/Nutritionist. Proc Xth Int Congr Dietetics, vol 1 and 2, John Libbey Eurotext, Londres

Pabst PE (1990) Les produits allégés. Option Qualité 76, 16-22

Picot D, Hellegouarch R, Claveau G (1986) La pratique de l'alimentation entérale. Cah Nutr Diét 21, 171-177

Prospérité Fermière (1989) Étude descriptive des ingrédients laitiers du marché européen.
Aliments infantiles, diététique grand public, nutrition entérale et parentérale, "pet foods"

Rafaitin M (1987) Produits de nutrition hospitalière et aliments de prescription. Mémoire de recherche. Univ Châtenay-Malabry

Schmidi MK, Massaro SS, Labuza TP (1988) Parenteral and enteral food systems. Food Technol 77-87

Wenner V (1978) Matières premières lactiques et modifications des produits laitiers traditionnels pour l'orientation de produits diététiques. $X X X^{e}$ Congr Int Lait, Paris 